\title{
Drug-coated balloon in superficial femoral artery in-stent restenosis
}

\author{
Donato Gerardi ${ }^{1}$, Arturo Alfani ${ }^{1}$, Tullio Tesorio² ${ }^{2}$ Angelo Cioppa ${ }^{2}$, Giovanni Esposito ${ }^{1}$, Eugenio Stabile ${ }^{1}$ \\ 1Division of Cardiology, Department of Advanced Biomedical Sciences, "Federico II" University, Napoli, Italy \\ 2Laboratory of Invasive Cardiology, Clinica Montevergine, Mercogliano, Italy
}

Adv Interv Cardiol 2018; 14, 1 (51): 9-14

DOI: https://doi.org/10.5114/aic.2018.74350

\begin{abstract}
A bstract
The femoropopliteal artery is one of the commonest sites of involvement in peripheral artery disease (PAD) leading to intermittent claudication and/or critical limb ischemia. Endovascular therapy for superficial femoral artery (SFA) disease has been recognized as a safe and efficient therapy and is recommended by current guidelines as the first-line approach. Although the widespread use of new-generation, self-expanding, nitinol stents in SFA stenosis has reduced the shortcomings associated with plain old balloon angioplasty (POBA), lumen renarrowing at the stented (in-stent restenosis - ISR) level still represents a relevant clinical problem, because of higher risk of recurrent ISR, occlusion and surgical revascularization compared to de-novo lesions. In this setting, different treatment options are available and drug-coated balloons (DCBs) have shown good results in terms of safety and effectiveness. In this review we examine the results of different trials exploring the outcome of using DCBs for the treatment of SFA ISR. The available data demonstrate that SFA ISR can be safely treated with percutaneous transluminal angioplasty with a DCB, with a reduction in recurrent restenosis and target lesion revascularization (TLR) at least at 1 year after POBA. The consistent and positive results of different registries and randomized trials support the use of DCB to reduce SFA ISR recurrence.
\end{abstract}

Key words: superficial femoral artery, superficial femoral artery, drug-coated balloon, drug-coated balloons, in-stent restenosis, in-stent restenosis.

\section{Introduction}

The femoropopliteal artery is one of the commonest sites of involvement in peripheral artery disease (PAD) leading to intermittent claudication and/or critical limb ischemia.

Endovascular therapy for superficial femoral artery (SFA) disease has been recognized as a safe and efficient therapy and is now recommended by current guidelines as the first-line approach, particularly in trans-Atlantic inter-society consensus (TASC) A-C lesions and in selected TASC D cases [1-3].

Standard approach consists in plain old balloon angioplasty (POBA) whereas stenting is considered only as a bailout treatment (for flow-limiting dissection, residual stenosis $>30 \%$ or elastic recoil). However, due to the increasing technical complexity of angioplasty cases, the use of stents is more frequently allowed, especially in the treatment of complex lesions (ulcerated, highly calcified plaques, long-segment stenosis/occlusions > $150 \mathrm{~mm}$ ) $[4,5]$.
Complex lesion percutaneous transluminal angioplasty (PTA) is associated with high rates of restenosis, reocclusion and symptom recurrence in up to $40-60 \%$ of patients in whom a standard balloon alone is used [6-8].

During balloon inflation an injury in the vascular wall is likely to be created, which can trigger subsequent biological processes. These comprise immediate elastic recoil of the arterial wall, intimal dissection, and negative vascular remodeling by neointimal hyperplasia. Stenting can prevent the elastic recoil and dissection and, thereafter, the risk of early occlusion. But stenting alone is not able to inhibit neointimal proliferation, which can even be stimulated by the stent struts. This overshooting biological response to vascular injury leads to loss of primary patency (PP), late lumen loss (LLL), occlusion, and the need for target lesion revascularization (TLR) $[9,10]$.

The use of self-expanding nitinol stents improved the patency rate of treated SFA but the occurrence of in-stent

\section{Corresponding author:}

Eugenio Stabile MD, Division of Cardiology, Department of Advanced Biomedical Sciences, "Federico II" University, Napoli, Italy, e-mail: gek050@hotmail.com

Received: 30.11.2017, accepted: 21.01.2018. 
restenosis (ISR) has become a considerable problem, occurring in up to $40 \%$ of patients within the first year [7, $11,12]$.

Nevertheless, stenting long femoro-popliteal (FP) segments with tubular nitinol stents has demonstrated a significant risk of stent fracture due to physiological torsion of the femoral artery, potentially resulting in either restenosis or acute thrombosis [13, 14].

\section{Classification of femoro-popliteal in-stent restenosis}

A classification system for FP ISR was proposed by Tosaka et al. [17] in which ISR lesions are assigned to one of three categories based on angiographic features:

- Class I (focal ISR): includes lesions $\leq 50 \mathrm{~mm}$ in length that are positioned in the stent body, at the stent edge, or both.

- Class II (diffuse ISR): includes stent body lesions and stent edge lesions $>50 \mathrm{~mm}$ in length.

- Class III: totally occluded ISR.

Recurrent ISR at 2 years after balloon angioplasty is higher in patients with class III lesions (84.9\%) than in those with class I or class II lesions (about 50\%). Recurrent occlusion at 2 years was $64.6 \%$ in class III compared with the other two classes ( $<20 \%)$.

Similarly, Armstrong et al. found that class III ISR remains an independent predictor of restenosis and reocclusion despite more frequent use of atherectomy and stent placement among patients with class III ISR [15-17].

\section{Endovascular treatment of superficial femoral artery in-stent restenosis}

Treatment of SFA-ISR is associated with increased risks of recurrent ISR, recurrent occlusion and surgical revascularization when compared to de-novo lesions [16]. Different treatment options are available, such as angioplasty with a balloon or stent. A plain balloon may expand a stent that was previously underdeployed or increase the luminal area through radial forces that compress the lesion outward against the stent. The high rate of immediate technical success after PTA for FP ISR followed by a similarly high rate of recurrence suggests that recoil of the hyperplastic lesion occurs frequently [17]. In the coronary territory, intravascular ultrasound (IVUS) studies demonstrated that angioplasty of the in-stent tissue causes its redistribution along the stent struts. This mechanism leads to high late lumen loss values during follow-up [18]. Therefore, outcomes may be closely related to the amount of in-stent tissue, as shown by Tosaka et al. [17]. Dick et al. [19] in 2008 reported $65 \%$ recurrent stenosis at 6 months in patients treated with POBA in long ISR lesions $(74 \pm 65 \mathrm{~mm})$.

\section{Drug-coated balloon (DCB) in superficial femoral artery in-stent restenosis}

Several studies have shown good results of DCBs in SFA in-stent restenosis. The potential safety and effectiveness of a therapeutic strategy based on the adjunctive use of DCB for the treatment of SFA-ISR was proposed by a single center Italian registry [20] including 39 consecutive patients who underwent conventional PTA for SFA-ISR and final post-dilation with paclitaxeleluting balloons (IN.PACT, Medtronic, Minneapolis, Minnesota). Technical success (defined as the ability to successfully perform PTA and DEB post-dilation with a residual stenosis $<30 \%$ ) and procedural success (defined as technical success without the occurrence of any in-hospital major adverse cardiac and cerebrovascular events) were achieved in $100 \%$ of patients. The primary endpoint (primary patency defined as proximal flow velocity ratio of 2.4 documented by duplex ultrasound at 12 months without target lesion revascularization) was obtained in $92.1 \%$ of patients.

Significant target lesion restenosis was observed only in 1 patient at 3 months and in 2 patients at 6 months. All these patients underwent a successful re-PTA. At 1 year secondary patency, clinical success (1 category improvement in the Rutherford scale from baseline or 2 categories if there was pre-existing tissue loss) and hemodynamic success (0.1 improvement in the ankle-brachial index during the period from baseline to post-procedure day 30 and no deterioration $>0.15$ from the maximum early post-procedure level at 1 year) were observed in $100 \%$ of patients [20]. From the same patients, a $70.3 \%$ primary patency rate at 2 years of follow-up was reported [21].

These study suggested that SFA-ISR can be safely treated with PTA with DCB, that the use of DCB is associated with low rates of recurrences and good clinical outcomes, and that class III ISR (occlusive) was not associated with an increased recurrence risk.

Similar results were reported from the PLAISIR study [22], which is a prospective and multicentre cohort study including 53 symptomatic patients with femoropopliteal in-stent restenosis (mean length: $86 \pm 32 \mathrm{~mm}$ ) treated by paclitaxel-eluting balloon angioplasty (In Pact Admiral, Medtronic, Santa Rosa, CA, USA). At 1 year freedom from TLR and target extremity revascularization (TER) were $90.2 \pm 4.2 \%$ and $85 \pm 5 \%$, respectively, and the primary patency rate was $83.7 \pm 5.0 \%$.

These data triggered the design of several clinical trials including the Drug-Eluting Balloon in Peripheral Intervention for In-Stent Restenosis (DEBATE-ISR) trial [23] of 44 patients with diabetes and FP-ISR treated with IN.PACT (Medtronic, Santa Rosa, CA, USA). Lesion length was 132 $\pm 86 \mathrm{~mm}$ in the DCB group vs. $137 \pm 82 \mathrm{~mm}$ in the POBA group. Recurrent restenosis occurred in $19.5 \%$ of patients in the DCB group vs. $71.8 \%$ in the POBA group. TLR was 
performed in $13.6 \%$ of patients in the DCB vs. $31 \%$ in the POBA group. However, at 3-year follow-up, the TLR rate in both groups was equivalent ( $40 \%$ vs. $43 \%$ ), thus suggesting that the use of DCB was only delaying the recurrence of restenosis. Of note, the presence of a class III ISR lesion was associated with a worse outcome in both groups [24].

The Femoral Artery In-Stent Restenosis (FAIR) trial [25] is a larger trial of DCBs for treatment of FP ISR in 119 patients (mean lesion length of $82.2 \pm 68.4 \mathrm{~mm}$ in both groups) including $28.6 \%$ chronic total in-stent occlusions of the SFA. The primary endpoint was the 6-month restenosis rate, which was in favor for the DCB when compared with POBA (15.4 vs. 44.7\%). At 1 year restenosis rates were 29.5 and $62.5 \%$, respectively and freedom from clinically driven TLR at 390 days was $90.8 \%$ and $52.6 \%$, respectively.

PACUBA is a prospective, dual-center, single-blind, randomized trial [26] of DCB angioplasty versus PTA in ISR of femoropopliteal arteries with blinded core laboratory adjudication, enrolling 74 patients. The mean lesion length was $173 \pm 113 \mathrm{~mm}$ in the DCB group and $184 \pm 88 \mathrm{~mm}$ in the POBA group. The 12-month primary patency rates were $40.7 \%$ versus $13.4 \%$ in the DEB versus POBA group. This finding was more evident in TASC $A$ and $B$ lesions.

$A$ recent meta-analysis of three prospective controlled trials comparing DCB versus POBA (DEBATE ISR, FAIR and PACUBA) for femoropopliteal ISR was performed by Wu et al. [27]. A total of 278 patients constituted the final study population, with 141 (50.7\%) patients treated by DCB and 137 (49.3\%) patients treated by POBA.

All the trials evaluated recurrent ISR at 12 months for DCB versus POBA. The incidence of combined recurrent ISR in the DCB group was significantly lower than that in the POBA group ( $34.8 \%$ vs. $73.1 \%$ respectively); consequently freedom from TLR in the DCB group was significantly higher than that in the POBA group $(82.2 \%$ vs. $54.1 \%)$.

No significant difference in the rate of major adverse events (MAEs) was found among the groups.

These data, even if limited by the few studies and patients enrolled, underline the superior efficacy outcome of DCB over POBA for the treatment of FP ISR.

The authors concluded that DCB is associated with improved clinical efficacy and a low incidence of side effects; however, it should be questioned whether POBA remains suitable as a comparator for FP ISR trials: recent research has shown that POBA is not an effective treatment strategy for FP ISR, especially for longer lesions, because of unacceptable results [28, 29].

The recently published Paclitaxel-Eluting Balloon Versus Conventional Balloon Angioplasty for In-Stent Restenosis of Superficial Femoral Artery (ISAR-PEBIS) 2-center trial [30] confirmed the good results of DCBs in SFA instent restenosis. In this trial 70 patients with symptom- atic in-stent restenosis were randomized to either $D C B$ or POBA (mean lesion length: $139 \pm 67 \mathrm{~mm}$ ), and roughly one third of lesions were completely occluded at the time of the index procedure.

At control angiography performed at 6 to 8 months, the percentage diameter stenosis ( $44 \pm 33 \%$ vs. $65 \pm 33 \%$ ) and binary restenosis were significantly reduced with DCB versus POBA (30\% vs. $59 \%$ ). At 24 -month follow-up, DCB was associated with a significant reduction of target lesion revascularization in comparison to POBA $(19 \%$ vs. 50\%). Other endpoints were analyzed (target vessel thrombosis, ipsilateral amputation, bypass surgery of the affected limb, and all-cause mortality at 24-month follow-up) but no difference was detected.

More recently, the data from the largest real world database on the use of DCB for the treatment of femoropopliteal obstructions have been reported.

The IN.PACT Global study is a prospective, multicenter, international, single arm clinical trial that evaluated the safety and effectiveness of a paclitaxel-coated DCB in a real-world population of 1535 patients with symptomatic (Rutherford class 2 to 4 ) femoropopliteal PAD (with single or multiple lesions; unilateral or bilateral disease; any lesion $2 \mathrm{~cm}$ or longer). A subset of 131 patients with long and complex de novo ISR was examined (mean lesion length $17.17 \pm 10.47 \mathrm{~cm}, 34 \%$ chronic total occlusion, $8.3 \%$ heavily calcified, $29 \%$ involving the proximal popliteal artery, 38.6\% with 0 -vessel runoff, and 35\% with diabetes). In this study an independent adjudication of adverse events and independent analysis of angiography and duplex ultrasonography were performed. Primary patency at 12 months was $88.7 \%$, with a clinically driven target lesion revascularization rate of $7.3 \%$. The primary safety outcome, a composite of freedom from device- and procedure-related mortality through 30 days and freedom from major target limb amputation and clinically driven TLR within 12 months, was $92.7 \%$. These data support DCB as desirable therapy in long, complex ISR [31, 32].

The Global SFA Registry is the first multicenter, worldwide, prospective, real-world study including 691 patients with stenosis or occlusion of a native femoropopliteal artery reporting a 24-month outcome for PAD patients treated with DCB. Freedom from TLR for all patients at 12 months was $93.4 \%$ and at 24 months was $89.3 \%$. In the subgroup of ISR patients ( $n=89$; target lesion length $154.4 \pm 97.1 \mathrm{~mm}$ ) freedom from TLR was $90.7 \%$ at 12 months and $84.6 \%$ at 24 months.

These data confirm DCB as a good option of treatment in real-world PAD patients, with a high burden of comorbidities and complex lesions, even in the ISR group, at 24-month follow-up [33].

A new drug-coated balloon catheter was evaluated in the AcoArt I randomized, multicenter, controlled clinical trial that compared efficacy and safety of an Orchid 
Table I. Peripheral drug-coated balloons available in the European Market [14]

\begin{tabular}{|c|c|c|c|c|c|}
\hline Brand name & Manufacturer & Excipient & $\begin{array}{c}\text { Paclitaxel } \\
\text { concentration }\left[\mu \mathrm{g} / \mathrm{mm}^{2}\right]\end{array}$ & Catheter type & $\begin{array}{c}\text { Guidewire } \\
\text { compatibility }\end{array}$ \\
\hline IN.PACT & Medtronic & Urea & 3.5 & OTW & $\begin{array}{l}0.014 " ; 0.018 " \\
0.035 "\end{array}$ \\
\hline $\begin{array}{l}\text { Lutonix } 14 \\
\text { Lutonix } 35 \\
\end{array}$ & Lutonix-bard & Polysorbate/sorbitol & 2 & OTW & 0.014"; 0.035" \\
\hline BIOPATH (prev. Freeway) & Eurocor/biosensor & Shellac & 3 & OTW & 0.014"; 0.035" \\
\hline Passeo Lux & Biotronik & BTHC & 3 & OTW & $0.018 ”$ \\
\hline Stellarex & Spectranetics & Polyethylene glycole & 2 & OTW & $0.035^{\prime \prime}$ \\
\hline Elutax sv & Aachen resonance & Dextane & 2.2 & RX/OTW & $0.014 " ; 0.018 "$ \\
\hline Legflow & Cardionovum & Shellac & 3 & RX/OTW & $0.014 " ; 0.035^{\prime \prime}$ \\
\hline Advance 18 ptx & Cook & None & 3 & OTW & $0.018 "$ \\
\hline Cotavance & Medtronic & Lopromide & 3 & RX/OTW & 0.014"; 0.035" \\
\hline Biopath & Biosensors & Shellac & 3 & OTW & 0.014"; $0.035^{\prime \prime}$ \\
\hline
\end{tabular}

OTW-over the wire, $R X$ - rapid exchange.

Table II. Comparison of published studies on DCB in superficial femoral artery in-stent restenosis

\begin{tabular}{|c|c|c|c|c|c|c|}
\hline Study & $\begin{array}{l}\text { Treatment } \\
\text { group }\end{array}$ & FU [months] & Cohort size & Lesion length [mm] & Freedom from TLR & Primary patency \\
\hline $\begin{array}{l}\text { Italian Registry } \\
{[20,21]}\end{array}$ & $\mathrm{DCB}$ & 24 & 39 & $82.9 \pm 78.9$ & $\begin{array}{l}89.5 \%(12 M) \\
78.4 \%(24 M)\end{array}$ & $\begin{array}{l}92.1 \%(12 M) \\
70.3 \%(24 M)\end{array}$ \\
\hline PLAISIR [22] & DCB & 12 & 53 & $86 \pm 32$ & $90.2 \%$ & $83.7 \%$ \\
\hline $\begin{array}{l}\text { DEBATE ISR } \\
{[23,24]}\end{array}$ & DCB vs. POBA & 36 & 44 vs. 42 & $132 \pm 86$ vs. $137 \pm 0.82$ & $\begin{array}{c}86.4 \% \text { vs. } 69 \%(12 \mathrm{M}) \\
60 \% \text { vs. } 57 \%(36 \mathrm{M})\end{array}$ & $\begin{array}{c}80.5 \% \text { vs. } 28.2 \% \\
(12 \mathrm{M})\end{array}$ \\
\hline FAIR [25] & DCB vs. POBA & 12 & 62 vs. 57 & $82.3 \pm 70.9$ vs. $81.1 \pm 66.2$ & $90.8 \%$ vs. $52.2 \%$ & $70.5 \%$ vs. $37.5 \%$ \\
\hline PACUBA [26] & DCB vs. POBA & 12 & 35 vs. 38 & $173 \pm 113$ vs. $184 \pm 88$ & $49 \%$ vs. $22.1 \%$ & $40.7 \%$ vs. $13.4 \%$ \\
\hline ISAR PEBIS [30] & DCB vs. POBA & 24 & 36 vs. 34 & $132 \pm 65$ vs. $146 \pm 69$ & $81 \%$ vs. $50 \%$ & $\begin{array}{l}70 \% \text { vs. } 41 \% \\
(6 \text { to } 8 \mathrm{M})\end{array}$ \\
\hline $\begin{array}{l}\text { IN.PACT Global } \\
\text { Study [31] }\end{array}$ & DCB & 12 & 131 & $171.7 \pm 104.7$ & $92.7 \%$ & $88.7 \%$ \\
\hline $\begin{array}{l}\text { Global SFA } \\
\text { registry [33] }\end{array}$ & DCB & 24 & 89 & $154.4 \pm 97.1$ & $\begin{array}{l}90.7 \%(12 M) \\
84.6 \%(24 M)\end{array}$ & $\begin{array}{c}83.3 \%(12 M) \\
66 \%(24 M)\end{array}$ \\
\hline
\end{tabular}

M-months.

paclitaxel-coated peripheral balloon catheter (Acotec Scientific, Beijing, China) with a standard uncoated balloon catheter in 200 patients (mean lesion length of $150 \mathrm{~mm}$; $25 \%$ in-stent restenosis, $55 \%$ occlusion or partial occlusion, $20 \%$ provisional stenting; $74 \%$ of patients in DCB group and $76 \%$ in uncoated balloon group had SFA lesion only, $15 \%$ and $11 \%$ respectively had popliteal artery combined with SFA lesions).

In the overall population, DCB showed better results in terms of late lumen loss at 6 months $(0.05 \pm 0.73 \mathrm{~mm}$ with DCB and $1.15 \pm 0.89 \mathrm{~mm}$ with uncoated balloons; $p<0.001)$ and restenosis $(2.5 \%$ and $70.8 \% ; p<0.001)$.

In the subgroup analysis, LLL in patients with ISR was $-0.04 \pm 0.69$ in the DCB group and $1.69 \pm 0.71$ with an uncoated balloon $(p<0.001)$, revealing a net luminal gain related to the paclitaxel effect of inhibiting the proliferation of smooth muscle cells and neointimal proliferation [34].

These results further confirm the efficacy of DCB and demonstrate the effective inhibition of restenosis following angioplasty with paclitaxel-coated balloons in a broad range of patients and lesions.

The peripheral DCB available in the European market (Table I) and a comparison of published trials on DCB use in ISR (Table II) are reported.

\section{Debulking and DCBs for the treatment of SFA ISR}

Laser atherectomy ablates and debulks neointimal tissue and suppresses platelet aggregation. The Photo-ablation Using the Turbo-Booster and Excimer Laser for In-Stent Restenosis Treatment (PATENT) [35] trial em- 
ployed the Turbo-Elite laser catheter and Turbo-Booster (Spectranetics, Colorado Springs, Colorado, USA) for FP ISR (mean lesion length, $123 \pm 95.9 \mathrm{~mm}$ ) in 90 patients. Adjunctive balloon angioplasty was performed in $87.8 \%$ of lesions. Primary patency at 6 months and 1 year was $64.1 \%$ and $37.8 \%$, respectively.

The Excimer Laser Randomized Controlled Study for Treatment of Femoropopliteal In-Stent Restenosis (EXCITE ISR) trial [36] randomized 250 patients to Turbo-Tandem (Spectranetics, Colorado Springs, Colorado, USA) laser atherectomy with PTA vs. PTA alone for the FP ISR. Mean lesion length was $196 \pm 120 \mathrm{~mm}$ with total occlusions in $30.5 \%$ and $36.8 \%$ of patients in the laser + PTA and PTA groups, respectively. Six-month freedom from TLR was 73.5\% (laser) vs. 51.8\% (PTA).

Only one study evaluated laser atherectomy with DEB in the treatment of in-stent restenosis. In 2014, Van den Berg et al. [37] showed a primary patency rate and a freedom from TLR rate of $86 \%$ at 18 months. This result suggested that laser atherectomy could assist DEB angioplasty effectively in the treatment of ISR. The ongoing Photoablative Atherectomy Followed by a Paclitaxel-Coated Balloon to Inhibit Restenosis in Instent Femoro-popliteal Obstructions (PHOTO-PAC) randomized trial will evaluate safety and efficacy of laser atherectomy before DCB angioplasty vs DCB angioplasty alone [38].

Directional atherectomy resects tissue with a cutting device in the longitudinal plane. There has been only one prospective study of this debulking modality for infrainguinal ISR. In a multicenter nonrandomized study of 40 lesions in 33 patients, primary patency at 1 year and 2 years was 33\% and 25\%, respectively [39]. However, the combination of directional atherectomy with DCB angioplasty offers the advantage of lesion debulking and the antirestenotic effect of DCB. In a retrospective analysis of 89 lesions in 29 patients with FP ISR treated with excisional atherectomy with DCB vs. excisional atherectomy with PTA, 1-year restenosis rates were $15.3 \%$ in the DCB group vs. $56.2 \%$ in the PTA group. Multivariable analysis for restenosis demonstrated that DCB treatment was independently associated with decreased restenosis compared with PTA [40].

Lesion preparation through an uncoated balloon before DCB angioplasty seems to be essential for calcified and complex lesions. The rationale behind combining $A R$ and DCBs is that removal of plaque facilitates local delivery of the antiproliferative drug and might therefore optimize drug delivery to the vessel wall [10].

\section{Conclusions}

The available data demonstrate that SFA ISR can be safely treated with PTA with DCB. Balloon angioplasty, which currently is the standard technique for ISR lesions, does not provide acceptable patency rates and performs even worse when an occlusive class III ISR lesion is treated. Randomized trials and large scale registry data demonstrate that DCB use, for the treatment of femoropopliteal ISR, is associated with superior efficacy outcomes compared with POBA at 1-year follow-up.

\section{Conflict of interest}

The authors declare no conflict of interest.

\section{References}

1. Fowkes FG, Rudan D, Rudan I, et al. Comparison of global estimates of prevalence and risk factors for peripheral artery disease in 2000 and 2010: a systematic review and analysis. Lancet 2013; 382: 1329-40.

2. Norgren L, Hiatt WR, Dormandy JA, et al. Inter-Society Consensus for the Management of Peripheral Arterial Disease (TASC II). J Vasc Surg 2007; 45 Suppl S: S5-67.

3. Rowe VL, Lee W, Weaver FA, et al. Patterns of treatment for peripheral arterial disease in the United States: 1996-2005. J Vasc Surg 2009; 49: 910-7.

4. Tendera M, Aboyans V, Bartelink ML, et al. ESC guidelines on the diagnosis and treatment of peripheral artery diseases: Document covering atherosclerotic disease of extracranial carotid and vertebral, mesenteric, renal, upper and lower extremity arteries: the Task Force on the Diagnosis and Treatment of Peripheral Artery Diseases of the European Society of Cardiology (ESC). Eur Heart J 2011; 32: 2851-906.

5. Katsanos K, Tepe G, Tsetis D, et al. Standards of practice for superficial femoral and popliteal artery angioplasty and stenting. Cardiovasc Intervent Radiol 2014; 37: 592-603.

6. Rocha-Singh KJ, Jaff MR, Crabtree TR, et al. Performance goals and endpoint assessments for clinical trials of femoropopliteal bare nitinol stents in patients with symptomatic peripheral arterial disease. Catheter Cardiovasc Interv 2007; 69: 910-9.

7. Schillinger M, Sabeti S, Loewe C, et al. Balloon angioplasty versus implantation of nitinol stents in the superficial femoral artery. N Engl J Med 2006; 354: 1879-88.

8. Mehrotra S, Paramasivam, Mishra S. Paclitaxel-coated balloon for femoropopliteal artery disease. Curr Cardiol Rep 2017; 19: 10.

9. Werk M, Albrecht T, Meyer DR, et al. Paclitaxel-coated balloons reduce restenosis after femoro-popliteal an gioplasty: evidence from the randomized PACIFIER trial. Circ Cardiovasc Interv 2012; 5: 831-40.

10. Herten M, Torsello G, Schönefeld E, et al. Drug-eluting balloons for femoropopliteal lesions show better performance in de novo stenosis or occlusion than in restenosis. J Vasc Surg 2015; 61: 394-9.

11. Soga Y, lida O, Hirano K, et al. Mid-term clinical outcome and predictors of vessel patency after femoropopliteal stenting with self-expandable nitinol stent. J Vasc Surg 2010; 52: 608-15.

12. Laird JR, Katzen BT, Scheinert D, et al. Nitinol stent implantation versus balloon angioplasty for lesions in the superficial femoral artery and proximal popliteal artery: twelve-month results from the RESILIENT randomized trial. Circ Cardiovasc Interv 2010; 3: 267-76.

13. Scheinert D, Scheinert S, Sax J, et al. Prevalence and clinical impact of stent fractures after femoropopliteal stenting. I Am Coll Cardiol 2005; 45: 312-5.

14. Cortese B, Granada J, Scheller B, et al. Drug-coated balloon treatment for lower extremity vascular disease intervention: 
an international positioning document. Eur Heart J 2016; 37 : 1096-103.

15. Armstrong EJ, Singh S, Singh GD, et al. Angiographic characteristics of femoropopliteal in-stent restenosis: association with long-term outcomes after endovascular intervention. Catheter Cardiovasc Interv 2013; 82: 1168-74.

16. Ho KJ, Owens C. Diagnosis, classification, and treatment of femoropopliteal artery in-stent restenosis. J Vasc Surg 2017; 65: 545-57.

17. Tosaka A, Soga Y, lida O, et al. Classification and clinical impact of restenosis after femoropopliteal stenting. J Am Coll Cardiol 2012; 59: 16-23.

18. Montorsi P, Galli S, Fabbiocchi F, et al. Randomized trial of conventional balloon angioplasty versus cutting balloon for in-stent restenosis. Acute and 24-hour angiographic and intravascular ultrasound changes and long-term follow-up. Ital Heart J 2004; 5: 271-9.

19. Dick P, Sabeti S, Mlekusch W, et al. Conventional balloon angioplasty versus peripheral cutting balloon angioplasty for treatment of femoropopliteal artery in-stent restenosis: initial experience. Radiology 2008; 248: 297-302.

20. Stabile S, Virga V, Salemme L, et al. Drug-eluting balloon for treatment of superficial femoral artery in-stent restenosis. J Am Coll Cardiol 2012; 60: 1739-42.

21. Virga V, Stabile E, Biamino G, et al. Drug-eluting balloons for the treatment of the superficial femoral artery in-stent restenosis: 2-year follow-up. JACC Cardiovasc Interv 2014; 7: 411-5.

22. Bague N, Julia P, Sauguet A, et al. Femoropopliteal in-stent restenosis repair: midterm outcomes after paclitaxel eluting balloon use (PLAISIR Trial). Eur J Vasc Endovasc Surg 2017; 53: 106-13.

23. Liistro F, Angioli P, Porto I, et al. Paclitaxel-eluting balloon vs. standard angioplasty to reduce recurrent restenosis in diabetic patients with in-stent restenosis of the superficial femoral and proximal popliteal arteries: the DEBATE-ISR study. J Endovasc Ther 2014; 21: 1-8.

24. Grotti S, Liistro F, Angioli P, et al. Paclitaxel-eluting balloon vs standard angioplasty to reduce restenosis in diabetic patients with in-stent restenosis of the superficial femoral and proximal popliteal arteries: three-year results of the DEBATE-ISR study. J Endovasc Ther 2016; 23: 52-7.

25. Krankenberg $H$, Tubler T, Ingwerson $M$, et al. Drug-coated balloon versus standard balloon for superficial femoral artery instent restenosis: the randomized femoral artery in-stent restenosis (FAIR) trial. Circulation 2015; 132: 2230-6.

26. Kinstner CM, Lammer J, Willfort-Ehringer A, et al. Paclitaxel-eluting balloon versus standard balloon angioplasty in in-stent restenosis of the superficial femoral and proximal popliteal artery: 1-year results of the PACUBA trial. JACC Cardiovasc Interv 2016; 9: 1386-92.

27. Wu R, Zilun L, Wang M, et al. Paclitaxel-coated versus uncoated balloon angioplasty for femoropopliteal artery in-stent restenosis. Int J Surg 2017; 42: 72-82.

28. Gray BH, Buchan JA. The treatment of superficial femoral artery in-stent restenosis: the jury is still out. JACC Cardiovasc Interv 2016; 9: 1393-6.

29. Zeller T, Rastan A, Macharzina R, et al. Drug-coated balloons: how should we incorporate into our practice in treating superficial femoral artery lesions? Curr Treat Options Cardiovasc Med 2015; 17: 380.

30. Ott I, Cassese S, Groha P, et al. ISAR-PEBIS (Paclitaxel-Eluting Balloon Versus Conventional Balloon Angioplasty for In-Stent
Restenosis of Superficial Femoral Artery): a randomized trial. J Am Heart Assoc 2017; 6: e006321.

31. Brodmann M, Keirse K, Scheinert D, et al. Drug-coated balloon treatment for femoropopliteal artery disease. The IN.PACT Global Study De Novo In-Stent Restenosis Imaging Cohort. JACC Cardiovasc Interv 2017; 10: 2113-23.

32. Niazi K, Tukaye D. Challenges of stent restenosis in superficial femoral and popliteal artery disease. JACC Cardiovasc Interv 2017; 10: 2124-5.

33. Thieme $M$, Von Bilderling P, Paetzel C, et al. The 24-month results of the Lutonix Global SFA Registry: worldwide experience with Lutonix drug-coated balloon. JACC Cardiovasc Interv 2017; 10: 1682-90.

34. Jia X, Zhang J, Zhuang B, et al. Acotec drug-coated balloon catheter randomized, multicenter, controlled clinical study in femoropopliteal arteries: evidence from the AcoArt I Trial. JACC Cardiovasc Interv 2016; 9: 1941-9.

35. Schmidt A, Zeller T, Sievert H, et al. Photoablation using the Turbo-Booster and excimer laser for in-stent restenosis treatment: twelve month results from the PATENT study. J Endovasc Ther 2014; 21: 52-60.

36. Dippel EJ, Makam P, Kovach R, et al. Randomized controlled study of excimer laser atherectomy for treatment of femoropopliteal in-stent restenosis: initial results from the EXCITE ISR trial (EXCImer Laser Randomized Controlled Study for Treatment of FemoropopliTEal In-Stent Restenosis). JACC Cardiovasc Interv 2015; 8: 92-101.

37. Van den Berg JC, Pedrotti M, Canevascini R, et al. In-stent restenosis: mid-term results of debulking using excimer laser and drug-eluting balloons: sustained benefit? J Invasive Cardiol 2014; 26: 333-7.

38. Photoablative atherectomy followed by a paclitaxel-coated balloon to inhibit restenosis in instent femoro-popliteal obstructions (PHOTOPAC). Available at: https://clinicaltrials.gov/ct2/ show/NCT01298947.

39. Beschorner U, Krankenberg H, Scheinert D, et al. Rotational and aspiration atherectomy for infrainguinal in-stent restenosis. Vasa 2013; 42: 127-33.

40. Sixt S, Carpio Cancino OG, Treszl A, et al. Drug-coated balloon angioplasty after directional atherectomy improves outcome in restenotic femoropopliteal arteries. J Vasc Surg 2013; 58: 682-6. 